\title{
An open software ecosystem for your everyday imaging task
}

\author{
Tobias Volkenandt, Torben Wulff, Sebastian Rhode and Martin Kuttge
}

Carl Zeiss Microscopy GmbH, Germany

When working with real world samples, you don't only have to become creative during sample preparation, mounting and acquisition, but also at a later stage during image analysis. Therefore, it is important to rely on a powerful and open software ecosystem offering the features and flexibility you need. In this contribution we will give an overview about the possibilities that come with the imaging and analysis suite ZEISS ZEN core.

The presented software ecosystem [1] was developed for industrial as well as academic customer groups in the field of materials science. It features a modular structure and can easily be extended with software packages for dedicated applications or analyses. The user can operate the software in two modes. The "free mode" provides access to all functionality and is perfectly suited for one-of evaluations or to develop new analysis routines specific for the sample at hand. In "job mode" the user is guided through a previously defined workflow and only sees the tools required for the current task. This is especially useful in industrial settings, where consistent and reproducible image analysis is key. Included functionality ranges from special acquisition workflows for light and scanning electron microscopy, over image processing, segmentation and analysis routines to flexible reporting and archiving options.

Here, we will highlight how automated measurement workflows can be adapted to a specific type of image or sample. These workflows can be customized to precisely derive the required quantitative information from an image, like grain or particles sizes, area coverage, orientation distribution, etc. Additionally, we will look at the necessary segmentation step in these workflows and show the advantages of machine learning for image segmentation and object classification. Implementations of correlative microscopy will also be covered since images from multiple modalities can significantly facilitate the analysis if a software offers ways to properly combine and align them. Finally, we will present a solution for even further extension and customization of the software ecosystem via including analysis routines and workflows from the cloud. Those can be coded by the community or completely self-made and thus bring highly specialized features to the robust and well-established suite.

All presented examples will be easy to understand and of practical use for any microscopist dealing with a wide variety of samples.

References

[1] https://www.zeiss.com/zen-core 